\title{
АНАЛИЗ ВОЗМОЖНОСТЕЙ СПУТНИКОВОГО МОНИТОРИНГА РАДИОАКТИВНОГО ЗАГРЯЗНЕНИЯ
}

\author{
В.О. Скрипачев, И.В. Суровцева, Ю.А. Полушковский, Д.Н. Чигрин
}

МИРЭА - Российский технологический университет (РТУ МИРЭА), пр. Вернадского, д. 78, Москва, Российская Федерация, 119454

E-mail: skripatchevv@inbox.ru, surovceva@mirea.ru, yapolu52@yandex.ru, chigrin@mirea.ru

В статье обоснована необходимость организации и ведения непрерывного глобального мониторинга радиоактивного загрязнения окружающей среды. Показаны отличительные особенности контактных и дистанционных способов наблюдения за изменениями в среде. Актуализированы разработки, внедрения эффективных методов дистанционного обнаружения и контроля радиоактивного загрязнения по диагностическим параметрам. Рассмотрены прямые и косвенные методы дистанционного мониторинга с учетом ионизирующего излучения. Проанализированы научные достижения в области спутникового мониторинга радиоактивного загрязнения окружающей среды. Предложена классификация перспективныхметодов мониторинга загрязнений с помощью технологических возможностей космических средств, включающая следующие методы: биоиндикационный, радиолокационные и СВЧ-радиометрия; методы регистрации флуоресценции в ультрафиолетовой области спектра, малых газовых составляющих, регистрации излучения нейтрального кластера и латентного тепла; обнаружение ионосферного отклика; метод «Поправка химического потенциала». Рассмотрены преимущества и недостатки спутниковой микроволновой СВЧ-радиометрии, а также особенности применения данного метода для решения тематических задач по запросам потребителей различных ведомств. Обрацено особое внимание на развитие и совериенствование малоизученных возможностей СВЧ-радиометрии. Обоснована необходимость диагностики изменений в приземных слоях атмосферы при радиоактивном загрязнении посредством реализации технологии СВЧ-радиометрии. При этом рекомендовано учитывать возможсности технических доработок приборов и осуществить коррекцию требований, предъявляемых к бортовому радиометру, комплексу приема и обработки целевой информации. Показана необходимость опережающего развития дистанционных методов с применением космических средств. Сделан вывод о целесообразности комплексирования разнородных методов, основанных на различных физических принципах, для повышения эффективности спутникового мониторинга радиоактивных загрязнений окружающей среды.

Ключевые слова: окружающая среда, радиоактивное загрязнение, спутниковый мониторинг, методы дистанционного мониторинга, СВЧ-радиометрия

\section{ANALYSIS OF SATELLITE MONITORING POSSIBILITIES RADIOACTIVE POLLUTION}

\author{
V.O. Skripachev, I.V. Surovceva, Yu.A. Polushkovskij, D.N. Chigrin
}

MIREA - Russian Technological University (RTU - MIREA), Vernadsky Av., 78, Moscow, Russian Federation, 119454

E-mail: skripatchevv@inbox.ru, surovceva@mirea.ru, yapolu52@yandex.ru, chigrin@mirea.ru

The article substantiates the necessity of organizing and conducting continuous global monitoring of radioactive pollution of the environment. The distinctive features of contact and re- 
mote methods of observing changes in the environment are shown. The developments, implementation of effective methods for remote detection and control of radioactive contamination by diagnostic parameters have been updated. Direct and indirect methods of remote monitoring taking into account ionizing radiation are considered. Scientific achievements in the field of satellite monitoring of radioactive pollution of the environment are analyzed. A classification of perspective methods for pollution monitoring using the technological capabilities of satellites are proposed, including the following methods: bioindication, radar and microwave radiometry; methods of registration of fluorescence in the ultraviolet region of the spectrum, small gas components, registration of radiation from a neutral cluster and latent heat; detection of ionospheric response; method "Correction of chemical potential". The advantages and disadvantages of satellite microwave microwave radiometry, as well as the features of the application of this method for solving thematic problems at the request of consumers of various departments, are considered. Special attention is paid to the development and improvement of the poorly studied capabilities of microwave radiometry. The necessity of diagnostics of changes in the surface layers of the atmosphere during radioactive pollution by means of the implementation of the technology of microwave radiometry has been substantiated. At the same time, it was recommended to take into account the possibilities of technical improvements of the devices and to correct the requirements for the on-board radiometer, the complex for receiving and processing target information. The necessity of the advanced development of remote sensing methods with the use of satellites are shown. It is concluded that it is advisable to combine heterogeneous methods based on different physical principles to improve the efficiency of satellite monitoring of radioactive pollution of the environment.

Key words: environment, radioactive pollution, satellite monitoring, remote sensing methods, microwave radiometry

Для цитирования:

Скрипачев В.О., Суровцева И.В., Полушковский Ю.А., Чигрин Д.Н. Анализ возможностей спутникового мониторингарадиоактивного загрязнения. Рос. хим. ж. (Ж. Рос. хим. об-ва). 2021. T. LXV. № 4. С. 25-30

For citation:

Skripachev V.O., Surovceva I.V., Polushkovskij Yu.A., Chigrin D.N. Analysis of satellite monitoring possibilities radioactive pollution. Ros. Khim. Zh. 2021. V. 65. N 4. P. 25-30

\section{ВВЕДЕНИЕ}

Проведенные в XX веке испытания ядерного оружия и ядерные взрывы, выполненные в промышленных целях, случившиеся техногенные аварии на объектах ядерного цикла, а также возрастание потенциальной угрозы применения ядерного оружия в современном мире обусловливают необходимость организации и ведения непрерывного глобального мониторинга радиоактивного загрязнения окружающей среды.

Способы наблюдения за различными изменениями в среде условно разделяют на контактные и дистанционные [1]. В отличие от контактных дистанционные способы, в т.ч. посредством спутниковых технологий, обеспечивают большую глобальность и оперативность наблюдения, что особенно важно в современных условиях, когда приоритетным считается заблаговременность упреждения принятия необходимых мер. Кроме того, одним из путей повышения эффективности монито- ринга радиоактивного загрязнения в условиях ресурсных или иных ограничений (пространственная удаленность, недоступность контактных измерений, недостаточность специальной техники и/или ограничение по времени) является применение дистанционных способов.

В связи с этим, все большую актуальность приобретает разработка и внедрение эффективных методов дистанционного обнаружения и контроля радиоактивного загрязнения окружающей среды по диагностическим параметрам, используя технологические возможности спутникового мониторинга [2].

Существующие методы дистанционного мониторинга подразделяют на прямые и косвенные. Первые регистрируют интенсивность и спектр ионизирующего излучения объекта, как правило, при непосредственном контакте измерительной техники с объектом измерения или при небольшом расстоянии (не более сотен метров). Вторые основаны на регистрации изменений в окружающей среде под действием ионизирующего излучения. 
При этом учитывается, что $\alpha$ - и $\beta$-излучения обладают сравнительно малой проникающей способностью, вследствие чего расстояние до объекта измерений сокращается. Таким образом, использование косвенных методов позволяет оценить уровень загрязнения по отклику окружающей среды на ионизирующее излучение, когда в качестве объектов среды принимаются приземные слои атмосферы, поверхности океана и суши.

\section{РЕЗУЛЬТАТЫ И ИХ ОБСУЖДЕНИЕ}

Согласно исследованиям, выполненным в Роскосмосе и Российской академии наук (РАН), наиболее перспективными методами мониторинга радиоактивных загрязнений из космоса признаны [3]:

Биоиндикаиионный метод, основанный на изменении цветности или флуоресценции приповерхностного слоя водоемов и растительного покрова, что может быть зарегистрировано бортовой аппаратурой спектрозональной съемки космического аппарата в диапазоне длин волн 320-370 нм. Однако этот метод обладает малой достоверностью и не дает однозначных результатов, что требует наземной проверки.

Метод дистанционной регистрации и измерения эффекта флуоресиенции атмосферного азота в ультрафиолетовой области спектра. Этот метод нашел реализацию в различных типах наземной специальной аппаратуры дистанционного обнаружения источников ионизирующих излучений. В большинстве случаев дальность действия метода ограничена несколькими десятков метров, что уменьшает возможность его применения. Однако эффект флуоресценции атмосферного азота, отчасти проявляемый при проведении спектрозональной съемки с борта космического аппарата, свидетельствует о возможности применения названного метода в качестве вспомогательного.

Метод дистаниионной регистрации малых газовых составляющих, основанный на выявлении ион-молекулярных реакций ионизированной атмосферы вследствие изменения концентрации ее основных малых газовых компонент (озон, гидроксил $\mathrm{OH}_{2}$ окислы азота $\mathrm{NO}$ и $\mathrm{NO}_{2}$ и др.). Реализация метода возможна со спутниковой орбиты, например, с помощью бортовых приборов газокорреляционной инфракрасной (ИК) радиометрии и лидаров. Измеренная таким методом концентрация малых газовых компонент отчетливо характеризует величину поглощенной дозы. Следует отметить, что метод дистанционной регистрации малых газовых составляющих обладает малой чувствительно- стью, особенно при относительно невысоких уровнях загрязнения.

Метод регистрации ионосферного отклика. Проведенные исследования показывают [3], что ионосфера чутко реагирует на процессы, происходящие в тропосфере и на поверхности Земли. Радиоактивное загрязнение приводит к локальной ионизации в тропосфере и как следствие изменению ее электрических характеристик. В силу анизотропной проводимости среды, связанной с геомагнитным полем, локальные неоднородности атмосферного электричества в тропосфере могут привести к появлению на уровне ионосферы горизонтальной составляющей компоненты электрического поля величиной порядка нескольких единиц мВ/м. Такие поля существенны для ионосферы и приводят к локальным изменениям электронной и ионной концентрации [4].

Таким образом, ионизация атмосферы приводит к изменению основных электрических характеристик в цепи тропосфера-ионосфера. Эти возмущения могут быть зарегистрированы с помощью ионозонда, размещенного на борту космического аппарата. Недостатками метода являются погрешности, возникающие от космического излучения и солнечных вспышек, а также учет не только природообусловленных суточных и сезонных вариаций параметров ионосферы, но и следствий известных антропогенных на нее воздействий $[5,6]$.

Метод регистрачии излучения нейтрального кластера. Во влажной атмосфере при определенных условиях могут образовываться сложные химически активные структуры типа ион-радикалов. В результате ассоциации таких гидратированных ион-радикалов образуется нейтральный кластер. Ожидаемый диапазон излучения кластеров перекрывается диапазонами, в которых работают теле- и радиопередатчики 108-142 МГц, 148-173 МГц. Это радиоизлучение может быть зарегистрировано специальным бортовым радиоспектрометром, к которому предъявляются высокие требования по чувствительности и избирательности.

Радиолокационные методы, основанные на регистрации и оценке радиоизлучения, отраженного от области повышенной ионизации. Наряду с другими методами мониторинга для диагностики локальных зон выбросов радиоактивных материалов радиолокационные методы основаны на регистрации сигнала от плазменных образований, образуемых ионизацией воздуха гамма-излучением от радиоактивной примеси факела выбросов в атмосферу радиационно-опасными предприятиями. Установлено, что отражение радиолокационного 
сигнала связано с сопутствующей радиации областью плазмообразования - плазмоидами, возникающими над зоной с объектами радиационных выбросов (например, вентиляционных труб АЭС) или поверхностных загрязнений (крыши зданий, могильники радиоактивных отходов, места осаждения радиоактивных выбросов и т.д.) в результате ионизации воздуха $\gamma$ - и $\beta$-излучением [4].

Следует отметить, что в основе радиолокационного метода лежат физические явления, сходные с радиометрическим методом, поскольку регистрируемые радиолокатором специфические эффекты подтверждают, что их природа связана с ионизационными образованиями [7].

Метод регистрации латентного тепла. Под воздействием ионизирующего излучения в приземном слое в зоне радиоактивного выброса может происходить локальное резкое падение влажности воздуха и повышение температуры. Это выделение тепла, называемое «латентным», вызывается нагревом паров воды радиоактивным излучением.

Метод мониторинга радиоактивного загрязнения посредством оценки выделения латентного тепла основан на процессе регистрации выделения скрытой теплоты испарения, который является следствием конденсации паров воды на ионах, образовавшихся после ионизации воздуха излучением радиоактивных загрязнений. В целях более достоверной их регистрации предлагается взаимное наложение карт зон загрязнений, полученных бортовой спутниковой аппаратурой в разных спектральных диапазонах. При этом для одной и той же местности, в одном масштабе создают два типа карт:

карты распределения латентного тепла в атмосфере, получаемые на основе анализа излучений в ИК-спектральном диапазоне 8-14 мкм;

карты распределения оценочных поправок к химическому потенциалу паров воды в атмосфере, полученные в результате измерений температуры и влажности в ее приземном слое на основе анализа радиоизлучений в диапазоне сантиметровых и миллиметровых волн, что в свою очередь способствует применению средств СВЧ-радиометрии.

Затем сравнивают данные по аномалиям к фону латентного тепла и аномалиям к фону оценочных поправок к химическому потенциалу паров воды. Места совпадения аномальных зон по обеим картам постулируют как места радиационных загрязнений.

На протяжении длительного времени применялся метод мониторинга радиоактивного загрязнения посредством оценки выделения латент- ного тепла, как аномалий термодинамических характеристик. Этот метод, разработанный К.А. Боярчуком с соавторами [3], применялся с использованием космических средств.

СВЧ-радиометрия. Перспективными методами обнаружения радиоактивных выбросов являются методы пассивной локации нейтральных и возбужденных атомов и молекул, появляющихся в атмосфере в результате радиоактивного облучения [8]. Один из методов пассивной локации - метод СВЧ-радиометрии, основанный на регистрации в микроволновом диапазоне электромагнитных волн отраженного, а при определенных условиях и рассеянного или собственного излучения от объектов наблюдения на поверхности земли и в атмосфере. В практике дистанционного зондирования Земли данный метод применяют более тридцати лет.

Метод «Поправка химического потенциала», реализация которого стала возможной благодаря установке отечественного СВЧ-радиометра МТВЗА-ГЯ на космических аппаратах «МетеорМ» №1 и №2.

В основе метода лежит регистрация вариаций термодинамических параметров воздуха и одновременного падения влажности под действием ионизирующего излучения газа радона в приземном слое. Измерение данных характеристик позволит получать информацию о распространении и уровне радиоактивных загрязнений. Однако, названный метод имеет существенный недостаток, связанный с коротким периодом полураспада $(3,8$ суток) основного изотопа радона ${ }^{222} \mathrm{Ra}$, а также с высокой вероятностью смены положения облака радона под действием ветра и диффузии [9].

Кроме того, применение СВЧ-радиометров важно для составления карт оценочных поправок к химическому потенциалу паров воды. При этом используют радиоизлучения в диапазонах частот 18,$7 ; 23,8 ; 36,5 ; 91 ; 183,31$ ГГц.

Методы, основанные на СВЧ-радиометрии, обладают рядом особенностей [10].

К преимуществам спутниковой микроволновой СВЧ-радиометрии относят: глобальность обзора поверхности Земли; регулярность съемки интересующих участков; независимость проведения съемки от времени суток; независимость проведения съемки от погодных условий.

Недостатки спутниковой СВЧ-радиометрии заключаются в следующем: низком пространственном разрешении; неустойчивой калибровке; завышенных требованиях к чувствительности радиометрических приемников. 
Особенностью метода СВЧ-радиометрии является низкое пространственное разрешение с высоты орбиты космического аппарата, составляющее порядка 15 км для диапазона 183 ГГц и до 150 км для диапазона 10,7 ГГц при диаметре зеркала антенны в пределах $60 \ldots 70$ см.

Технология спутниковой СВЧ-радиометрии создавалась для решения задач гидрометеорологии, поэтому приведенные численные значения пространственного разрешения в основном удовлетворяют потребителей метеоинформации и не подходят для непосредственного решения задач диагностики радиоактивного заражения. Такие значения пространственного разрешения недостаточны для мониторинга радиоактивного загрязнения, когда размеры зон заражения на местности и, как следствие, размеры областей проявления маркеров ионизации варьируются в пределах от десятков метров до нескольких километров. Поэтому в целях повышения пространственного разрешения данного метода разработчики СВЧ-радиометров стремятся к увеличению диаметра антенн до 2 м и более, что ограничено массово-габаритными требованиями конструкции космического аппарата.

СВЧ-радиометрия при своей многолетней истории применения на текущий момент времени не имеет большого опыта в интересах спутникового мониторинга радиоактивного загрязнения окружающей среды. Недостаточно изучены участки

\section{Л И ТЕР А Т УР А}

1. Экологический мониторинг атмосферы: практикум для бакалавров НП 20.03.01 «Техносферная безопасность» по профилю «Инженерная защита окружающей среды» / сост. Е.Н. Калюкова. - Ульяновск: УлГТУ. 2015. 131 с.

2. Суровцева И.В. Технологии дистанционного мониторинга окружающей среды при определенных экстремальных ситуациях. Рос. хим. ж. (Ж. Рос. хим. об-ва им. Д.И. Менделеева). 2020. T. LXIV. № 4. С. 97-103. DOI: 10.6060/rcj.2020644.14.

3. Пулинеи С.А., Хегай В.В., Боярчук К.А., Ломоносов А.М. Атмосферное электрическое поле как источник изменчивости ионосферы. УФН. 1998. Т. 168. № 5. С. 582-589. DOI: $10.3367 / \mathrm{UFNr} .0168 .199805 \mathrm{~h} .0582$.

4. Boyarchuk K.A., Lomonosov A.M., Pulinets S.A., Hegai V.V. Ionosphere respons on the radioactive pollution in troposphere. The new remote sensing method. - BRAS Physics / Supplement, Physics of Vibrations. 1997. V. 61. N 4. P. 260-266.

5. Суровцева И.В. Технологии приёма, обработки и распределения геофизической информации / XIV Всероссийская научная конференция «Технологии и материалы для экстремальных условий», Туапсе, Россия (16-20 сентября 2019). М.: МЦАИ РАН. 2019. С. 272-280. DOI: 10.26103/MZ2019.43.25.039. спектрального диапазона, позволяющие достоверно определять границы зон и концентрацию малых газовых составляющих атмосферы, свидетельствующих о масштабах загрязнения. Кроме того, требования к информативности данного метода определяют необходимость освоения более высоких частотных диапазонов (от 2 ТГц), что сопряжено с технологическими сложностями в создании высокочастотных элементов радиотракта $[11,12]$. В этом случае приходится учитывать не только частотные участки электромагнитного излучения (ЭМИ) в «окнах прозрачности» атмосферы, но и частоты отражения ЭМИ исследуемых объектов на поверхности Земли.

\section{ВЫВОДЫ}

На основании вышеизложенного можно резюмировать, что комплексирование двух и более методов мониторинга радиоактивного загрязнения и реализующих их технических устройств, работа которых основана на различных физических принципах, обуславливает проявление эффекта синергизма. Авторы полагают целесообразным объединение усилий исследователей и научных групп для решения широкого спектра задач посредством применения технологии СВЧ-радиометрии, а также комплексирования известных методов в целях эффективности мониторинга радиоактивного загрязнения окружающей среды.

\section{REFERENCES}

1. Environmental monitoring of the atmosphere: a workshop for bachelors of NP 20.03.01 "Technosphere safety" in the profile "Engineering environmental protection" / sost. E.N. Kalyukova. - Ul'yanovsk: UlGTU. 2015.131 p.

2. Surovceva I.V. Technologies for remote monitoring of the environment in certain extreme situations. Ros. xim. zh. (Zh. Ros. xim. ob-va im. D.I. Mendeleeva). 2020. V. LXIV. N 4. P. 97-103. DOI:10.6060/rcj.2020644.14.

3. Pulinets S.A., Hegaj V.V., Boyarchuk K.A., Lomonosov A.M. Atmospheric electric field as a source of ionospheric variability. UFN. 1998. V. 168. N 5. P. 582-589. DOI: 10.3367/UFNr.0168.199805h.0582.

4. Boyarchuk K.A., Lomonosov A.M., Pulinets S.A., Hegai V.V. Ionosphere respons on the radioactive pollution in troposphere. The new remote sensing method. - BRAS Physics / Supplement, Physics of Vibrations. 1997. V. 61. N 4. P. 260-266.

5. Surovceva I.V. Technologies for receiving, processing and distributing geophysical information / XIV Vserossijskaya nauchnaya konferenciya «Texnologii i materialy` dlya e`kstremal'ny`x uslovij», Tuapse, Rossiya (16-20 sentyabrya 2019). M.: MCzAI RAN. 2019. P. 272-280. DOI:10.26103/MZ2019.43.25.039. 
6. Kaloshin I., Kuznetsov V., Skripachev V., Surovceva I. Capabilities evaluation of spaceborne scientific equipment for geophysical applications / MATEC Web of Conferences 102, 01024 (2017) V International Forum for Young Scientists "Space Engineering". DOI: 10.1051/matecconf/201710201024.

7. Боярчук К.А., Карелин А.В., Макриденко Л.А. Перспективы мониторинга из космоса радиоактивных загрязнений на поверхности Земли и в нижних слоях атмосферы. Вопросы электромеханики. 2005. Т. 102. С. 183-209.

8. Исакова А.И., Пенин С.Т. Программное обеспечение системы обработки данных СВЧ-радиометра для прогноза загрязнения атмосферы радиоактивными выбросами. Доклады ТУСУРа. № 1 (19). Часть 1. 2009. С. 82-87.

9. Копытин Ю.Д., Носов В.В., Антипов А.Б., Исакова А.И., Самохвалов М.А., Чистякова Л.К. Дистанционные методы прогноза нефтяных, рудных и техногенных аномалий по геоатмосферным проявлениям. - Томск: Изд. ИОА СО РАН. 2000. $314 \mathrm{c}$.

10. Михайлов В.Ф., Брагин И.В., Брагин С.И. Микроволновая спутниковая аппаратура дистанционного зондирования Земли: Учеб. пособие / СПбГУАП. СПб., 2003. 404 с.

11. Ulaby F. Microwave Radar and Radiometric Remote Sensing; The University of Michigan Press: Chicago, IL, USA; Ann Arbor. MI. USA. 2014. 1116 p.

12. Fuzhong Weng. Passive Microwave Remote Sensing of the Earth: for Meteorological Applications. Wiley-VCH. 2018. $384 \mathrm{p}$.
6. Kaloshin I., Kuznetsov V., Skripachev V., Surovceva I. Capabilities evaluation of spaceborne scientific equipment for geophysical applications / MATEC Web of Conferences 102, 01024 (2017) V International Forum for Young Scientists "Space Engineering". DOI: 10.1051/matecconf/201710201024.

7. Boyarchuk K.A., Karelin A.V., Makridenko L.A. Prospects for monitoring from space of radioactive contamination on the Earth's surface and in the lower layers of the atmosphere. Voprosy` e`lektromexaniki. 2005. V. 102. P. 183-209.

8. Isakova A.I., Penin S.T. Software of the microwave radiometer data processing system for predicting atmospheric pollution by radioactive emissions. Doklady` TUSURa. 2009. № 1(19). chast' 1. P. 82-87.

9. Kopy`in Yu.D., Nosov V.V., Antipov A.B., Isakova A.I., Samoxvalov M.A., Chistyakova L.K. Remote methods for predicting oil, ore and man-made anomalies by geoatmospheric manifestations - Tomsk: Izd. IOA SO RAN. 2000. 314 p.

10. Mihailov V.F., Bragin I.V., Bragin C.I. Microwave satellite equipment for remote sensing of the Earth: The textbook / SPbGUAP. SPb. 2003. 404 p.

11. Ulaby F. Microwave Radar and Radiometric Remote Sensing; The University of Michigan Press: Chicago, IL, USA; Ann Arbor. MI. USA. 2014. 1116 p.

12. Fuzhong Weng. Passive Microwave Remote Sensing of the Earth: for Meteorological Applications. Wiley-VCH. 2018. $384 \mathrm{p}$.

Поступила в редакичию (Received) 01.08.2021

Принята к опубликованию (Accepted) 03.09.2021 